\title{
Tailored pyroresistive performance and flexibility by introducing a secondary thermoplastic elastomeric phase into graphene nanoplatelet (GNP) filled polymer composites for self-regulating heating devices
}

Yi Liu, ${ }^{\text {a,c }}$ Han Zhang, ${ }^{\text {a,c, } *}$ Harshit Porwal,,${ }^{\text {a,c }}$ Wei Tu, ${ }^{\text {c }}$ Kening Wan, ${ }^{\text {a Jamie Evans, }}{ }^{\mathrm{b}}$ Mark Newton, ${ }^{\mathrm{b}}$ James J.C. Busfield, ${ }^{\mathrm{a}}$ Ton Peijs, ${ }^{\mathrm{a}, \mathrm{c}}$ and Emiliano Bilotti ${ }^{\mathrm{a}, \mathrm{c}, *}$

a. School of Engineering and Materials Science, Queen Mary University of London, Mile End Road, London E1 4NS, UK, E-mail: han.zhang@qmul.ac.uk; e.bilotti@qmul.ac.uk;

b. LMK Thermosafe Ltd., 9-10 Moonhall Business Park, Helions Bumpstead Rd, Haverhill, Suffolk CB9 7AA, UK

c. Nanoforce Technology Ltd., Joseph Priestley Building, Queen Mary University of London, Mile End Road, E1 4NS London, UK

\begin{abstract}
Flexible and controllable self-regulating heating devices have great potential for use in applications such as healthcare devices, soft robotics, artificial skins and wearable electronics. Conventional self-regulating heating devices are often limited by the rigid nature of the polymer matrices, particularly at high conductive filler concentrations. In this paper, this limitation has been successfully tackled by using binary polymer blends that can achieve a desirable combination of mechanical, electrical and pyroresistive properties. The addition of a suitable secondary thermoplastic elastomeric polymeric phase did not only improve material flexibility, but did also tune the positive temperature coefficient (PTC) behaviour. For the first time, we systematically explore the effect of different blend morphologies as well as the selective localization of conductive fillers like graphene nanoplatelets (GNPs) on the overall mechanical and pyroresistive performance of self-regulating conductive polymer composites (CPCs). The effect of different blend morphologies was studied using different thermoplastic
\end{abstract}


elastomers (TPEs) as secondary phases, and various blend compositions, into a GNP filled high density polyethylene (HDPE) nanocomposite. Blend morphologies included immiscible binary blends with a fine and coarse droplet morphology and a co-continuous morphology. In doing so, this study serves as a guideline for the selection of a secondary elastomeric phase in polymer blend based CPCs for optimised device flexibility and self-regulating heating functions.

\section{Introduction}

Conductive polymer composites (CPCs) have attracted increasing amounts of attention due to their ease of fabrication and wide range of applications. ${ }^{1-3}$ Many novel devices in the area of health monitoring and wearable electronics are made of CPCs, with various functionalities including sensitivity to strain, damage, humidity, and temperature. ${ }^{4-9}$ Self-regulating heating devices benefit from another intrinsic feature found in CPCs: the positive temperature coefficient (PTC) effect, where the electrical resistivity increases with increasing operating temperature. The large increment in resistance of these materials is in correspondence with a phase transition of the polymer matrix and results in an automated safety cut-off of the heating process in the device. ${ }^{10,11}$ This temperature induced phenomenon has also been used in other applications, like temperature sensors, current limiting devices, resettable fused and safety batteries.

Since the initial discovery of large PTC behaviour of CPCs in the 1970s, high density polyethylene (HDPE) is one of the most used polymeric materials for self-regulating heating applications. $^{10,12}$ The semi-crystalline nature, good thermal stability and high thermal expansion rate around the melting point provide HDPE-based CPCs with superior PTC behaviour. ${ }^{13-15}$ However, the limited mechanical ductility, particularly in the case of high conductive filler loadings, makes these composites unsuitable for a number of applications that require greater flexibility. ${ }^{16,17}$ 
Many attempts have been made to improve the flexibility and reduce the brittleness of polymer based self-regulating heating materials. For example, one approach is to utilise high aspectratio nanofillers such as carbon nanotubes (CNTs) and graphene instead of traditional carbon black (CB) to lower the overall filler loading. ${ }^{18-21}$ However, the negative temperature coefficient (NTC) effect, where the resistance decreases with increasing temperature, may dominate the behaviour of these composites, particularly for nanoparticles which undergo extensive agglomeration or flocculation. Other methods involve the use of more flexible polymeric matrices like rubbers or thermoplastic elastomers (TPEs). ${ }^{22}$ Unfortunately, in the latter case, the PTC performance is often sacrificed due to the absence or a low degree of crystallinity and the limited thermo-mechanical response. ${ }^{9}$

In the current study, unlike traditional PTC devices which typically employ only a single polymer for the CPC, binary blends of HDPE and a TPE are pursued to find an optimal balance between ductility or flexibility and the PTC effect. Although PTC materials based on immiscible polymer blends have been previously investigated, the main focus has been on the elimination of the NTC effect and improvement of the PTC reproducibility, as reported by Zha et al. and Chen et al. ${ }^{20,23}$ However, the addition of an elastomeric phase into a rigid polymer based CPC to increase the ductility and flexibility while preserving the pyroresistive properties has not yet been systematically studied. This will be the aim of this paper.

The properties of multiphase polymer composites areguided by many factors, including the location of the filler, the compatibility between the phases, and their morphologies. ${ }^{13,24-26}$ The location of nanoparticles in immiscible polymer blends prepared by melt mixing is the result of a complex interplay between various thermodynamical and processing parameters, such as the absolute viscosity and viscosity ratio of the polymers, interfacial tension of the constituents, and compounding procedures. ${ }^{27}$ The electrical conductivity of multiphase CPCs, in particular, is influenced by the conductive filler content in the filler-rich phase, and the continuity of this 
phase in the blends. ${ }^{28,29}$ The mechanism of the heterogeneous distribution of fillers is explained by the difference in the affinity of fillers to each component of the polymer blend. ${ }^{30}$ By using such concepts, the filler location can be predicted to some extent.

The objective of this study is to explore the mechanism of the conductive network formation in binary CPC systems, and identify the influence of a secondary elastomeric phase on the PTC behaviour. For this, three thermoplastic elastomers (TPEs); styrene-ethylene-butylene-styrene (SEBS), ethylene-propylene rubber (EPR), and thermoplastic polyurethane (TPU), were selected and melt blended into a graphene nanoplatelet (GNP) filled HDPE masterbatch. These blend systems were selected as they should produce three distinct types of blend morphologies.

These three types of morphologies are: (i) an immiscible polymer blend with a very fine droplet morphology for the SEBS/HDPE blends. SEBS is a triblock copolymer containing styrene blocks and ethylene/butylene blocks and has been widely used as a compatibilizer for polystyrene/polyethylene blends. ${ }^{31,32}$ Blended with HDPE it can form a fine droplet morphology. (ii) An immiscible polymer blend with a co-continuous morphology for the EPR/HDPE blends at around 50/50 volume ratio due to the ethylene blocks in EPR. ${ }^{33}$ (iii) An immiscible polymer blend with a coarse droplet morphology for the TPU/HDPE system, due to the large differences in polarities and high interfacial tensions. ${ }^{34,35}$

This paper reveals for the first time the influence of different blend morphologies as well as selective localization of conductive nanofillers (GNPs) on the overall mechanical and pyroresistive performance of self-regulating CPCs. In doing so, it serves as a guideline for the selection of a secondary phase in polymer blend-based CPCs for the optimisation of both device flexibility and self-regulating heating performance. (This work is protected by International Patent Application. $)^{36}$

\section{Experimental}




\section{Materials}

A list of polymers and conductive fillers used in this work is presented in Table 1. All polymers were dried at $80{ }^{\circ} \mathrm{C}$ for 12 hours, prior to processing.

Table 1 List of polymers and conductive fillers

\begin{tabular}{|c|c|c|c|c|}
\hline Materials & Chemical formula & supplier & $\begin{array}{l}\text { Trade } \\
\text { name }\end{array}$ & $\begin{array}{l}\text { Information } \\
\text { (from datasheet) }\end{array}$ \\
\hline $\begin{array}{l}\text { High density } \\
\text { polyethylene }\end{array}$ & & INEOS & $\begin{array}{l}\text { HD521 } \\
8 \mathrm{EA}\end{array}$ & $\begin{array}{l}\text { Density of } 0.952 \\
\mathrm{~g} / \mathrm{cm}^{3} \text {, and melt }\end{array}$ \\
\hline $\begin{array}{l}\text { Styrene- } \\
\text { ethylene- } \\
\text { butylene- } \\
\text { styrene (SEBS) }\end{array}$ & & Kraton & FG1901 & $\begin{array}{l}\text { Polystyrene } \\
\text { content of } 30 \% \\
\text { and density of } \\
0.91 \mathrm{~g} / \mathrm{cm}^{3}\end{array}$ \\
\hline $\begin{array}{l}\text { Ehylene- } \\
\text { propylene } \\
\text { rubber (EPR) }\end{array}$ & & Dow & $\begin{array}{l}\text { Versify } \\
\text { TM } 2200\end{array}$ & $\begin{array}{l}\text { Density of } 0.876 \\
\mathrm{~g} / \mathrm{cm}^{3}\end{array}$ \\
\hline $\begin{array}{l}\text { Thermoplastic } \\
\text { polyurethane } \\
\text { (TPU) }\end{array}$ & & Lubrizol & $\begin{array}{l}\text { Estane }^{\circledR} \\
58437\end{array}$ & $\begin{array}{l}\text { Density of } 1.19 \\
\mathrm{~g} / \mathrm{cm}^{3}\end{array}$ \\
\hline $\begin{array}{l}\text { Graphene } \\
\text { nanoplatelets } \\
\text { (GNPs) }\end{array}$ & & $\begin{array}{l}X G \\
\text { Science }\end{array}$ & $\begin{array}{l}x G n P^{\circledast} \\
\text { M15 }\end{array}$ & $\begin{array}{l}\text { Average diameter } \\
\text { of } 15 \mu \mathrm{m} \text {, surface } \\
\text { area of } 150 \mathrm{~m}^{2} / \mathrm{g} \text {, } \\
\text { and density of } 2.2 \\
\mathrm{~g} / \mathrm{cm}^{3}\end{array}$ \\
\hline
\end{tabular}

\section{Fabrication of composites}

Pure immiscible blends (SEBS/HDPE, EPR/HDPE and TPU/HDPE) of different compositions $(10 / 90,30 / 70,50 / 50,70 / 30$ and 90/10 v/v) were prepared by melt mixing using a DSM X'plore 15 micro-compounder (The Netherland), at $200 \mathrm{1C}$ and $50 \mathrm{rpm}$ for $5 \mathrm{~min}$. Graphene nanoplatelets, xGnPs M15 from XG Science (USA), were compounded at a loading of $24 \mathrm{wt} \%$ 
with HDPE using a Dr Collin (Germany) twin-screw compounder (ZK25 × 32 D). Throughput was $2 \mathrm{~kg} \mathrm{~h}^{-1}$ using a screw speed of $220 \mathrm{rpm}$ and a temperature varying from $190{ }^{\circ} \mathrm{C}$ to $240{ }^{\circ} \mathrm{C}$, over 8 heating zones. The produced HDPE/GNP (24 wt $\%$ ) compound was used as a masterbatch (MB) and diluted with the different thermoplastic elastomers (TPEs) to desired concentrations using the DSM X'plore 15 micro-compounder and the same mild processing conditions as used for the neat immiscible blends. The extruded strands that were produced were then cut into pellets and compression moulded into rectangular shaped samples with dimensions of $30 \mathrm{~mm} \times 10 \mathrm{~mm} \times 2 \mathrm{~mm}$, using a Dr Collin hot press P300E, at $2201 \mathrm{C}$ for 5 min and 60 bar pressure. A copper mesh $(0.26 \mathrm{~mm}$ aperture and $0.16 \mathrm{~mm}$ wire diameter $)$ was embedded on both sides of the sample during compression moulding and used as an electrode. A list of all the blends and composites produced is presented in Table 2.

Table 2 Compositions and code names of the samples

\begin{tabular}{|c|c|c|c|}
\hline \multirow[t]{2}{*}{ Composites } & & \multicolumn{2}{|c|}{ Composition } \\
\hline & & Weight fractions & Volume fractions (equivalent) \\
\hline \multirow[t]{5}{*}{ HDPE/GNP } & & 12 wt. \% GNP + HDPE & 5.6 vol. $\%$ GNP + HDPE \\
\hline & & 15 wt. \% GNP + HDPE & 7.0 vol. $\%$ GNP + HDPE \\
\hline & & 18 wt. \% GNP + HDPE & 8.7 vol. $\%$ GNP + HDPE \\
\hline & & 22 wt. \% GNP + HDPE & 10.8 vol.\% GNP + HDPE \\
\hline & $\begin{array}{l}\text { Masterbatch } \\
\text { (MB) }\end{array}$ & 24 wt.\% GNP + HDPE & 12.0 vol. $\%$ GNP + HDPE \\
\hline \multirow[t]{4}{*}{ SEBS/HDPE/GNP } & & 10 wt. $\%$ SEBS + MB & 11.9 vol. $\%$ SEBS + MB \\
\hline & & 20 wt. $\%$ SEBS + MB & 23.2 vol. $\%$ SEBS + MB \\
\hline & & 35 wt. $\%$ SEBS + MB & 39.5 vol. $\%$ SEBS + MB \\
\hline & & 50 wt. $\%$ SEBS + MB & 54.8 vol. $\%$ SEBS + MB \\
\hline \multirow[t]{4}{*}{ EPR/HDPE/GNP } & & $10 w t . \%$ EPR + MB & 12.4 vol. \% EPR + MB \\
\hline & & $20 w t . \%$ EPR + MB & 24.1 vol. \% EPR + MB \\
\hline & & $35 w t . \%$ EPR + MB & 40.7 vol. $\%$ EPR + MB \\
\hline & & 50 wt. \% EPR + MB & 56.0 vol. $\%$ EPR + MB \\
\hline \multirow[t]{4}{*}{ TPU/HDPE/GNP } & & $10 w t . \%$ TPU + MB & 9.3 vol.\% TPU + MB \\
\hline & & 20 wt. \% TPU + MB & 18.8 vol. $\%$ TPU + MB \\
\hline & & 35 wt. \% TPU + MB & 33.3 vol. $\%$ TPU + MB \\
\hline & & 50 wt. $\%$ TPU + MB & 48.1 vol. $\%$ TPU + MB \\
\hline
\end{tabular}




\section{Characterisation}

Scanning electron microscopy (SEM) (FEI Inspector-F, Netherlands) was used to image the blend morphology as well as the distribution or selective localization of the GNPs in the blends. The micro-structure of the blend composites was studied on cold fractured surfaces, which were induced by immersing the specimens into the liquid nitrogen for $10 \mathrm{~min}$. All the surfaces analysed were gold sputtered before imaging.

A drop shape analyser (DSA100, KRUSS GmbH, Germany) was used to measure the contact angle between a reference liquid (water and ethylene glycol) droplet and solid polymer films. Surface energies were calculated from the contact angle data of sessile drops of $5 \mathrm{~mL}$.

The pyroresistive behaviour of all samples was tested with an apparatus consisting of a temperature controlled oven (heating rate of $2{ }^{\circ} \mathrm{C} \min ^{-1}$ ) and a two-point resistance measurement unit, obtained by combining a picoammeter (Keithley 6485, USA) with a DC voltage source (Agilent HP 6614C, USA). The thermocouple was placed close to, but not touching, the specimen to ensure accurate reading. A constant voltage $(1 \mathrm{~V})$ was applied during heating and cooling cycles on the rectangular specimens while the current and temperature were monitored and recorded simultaneously.

To evaluate the Joule heating behaviour of the specimens, alternating voltage was applied to the sample whilst a thermal infrared camera (FLIR E40, UK) was used to record temperature changes and to capture thermal images during heating.

The mechanical behaviour of the composite materials was evaluated using bending tests and tensile tests. Dumbbell shaped tensile specimens (according to ISO 37 type 4) were tested using an Instron 5586 at room temperature (RT), equipped with a $100 \mathrm{~N}$ load cell, at a rate of $0.5 \mathrm{~mm}$ $\min ^{-1} \cdot{ }^{37}$ Three-point bending was used to evaluate flexural behaviour on rectangular shaped samples, with the electrical resistance measured upon bending. 


\section{Results and discussion}

\section{Polymer blend morphologies}

The phase behaviour of multi-component polymeric systems is of crucial importance for determining their physical properties. In the case of immiscible blends, forming separate phases, important issues are the phase morphology (size and shape) and the nature of compatibility (wetting and adhesion) between the phases. SEM micrographs of cryo-fracture surfaces of the three immiscible polymer blends with different TPE/HDPE composition volume ratios (10/90, 30/70, 50/50, 70/30 and 90/10 v/v) are shown in Fig. 1a, which are SEBS/HDPE, EPR/HDPE, and TPU/HDPE, respectively. All the three types of polymer blends have a phase inversion taken place between 30/70 and 70/30 v/v observed from the SEM images. SEBS/HDPE blends show a morphology of finely dispersed droplets of SEBS around 50/50 v/v in a HDPE matrix due to a good compatibility for all compositions. This fine morphology is due to the SEBS triblock copolymer's chemical structure, which is highly compatible with polyethylene, and is well studied by a number of researchers..$^{32,38,39}$ The 50/50 v/v EPR/HDPE blend shows a cocontinuous morphology with the two phases clearly identifiable. The 50/50 v/v TPU/HDPE blends on the other hand exhibit an 'island-in-the-sea' morphology of coarse TPU droplets in HDPE. Fig. $1 \mathrm{~b}$ illustrates the most representative three types of blend morphologies at 50/50 v/v, which are (i) immiscible binary blend with a very fine droplet-like morphology; (ii) immiscible co-continuous binary blend; and (iii) immiscible 'island-in-the-sea' binary blend with a coarse droplet morphology consisting of a continuous phase and a dispersed phase. 

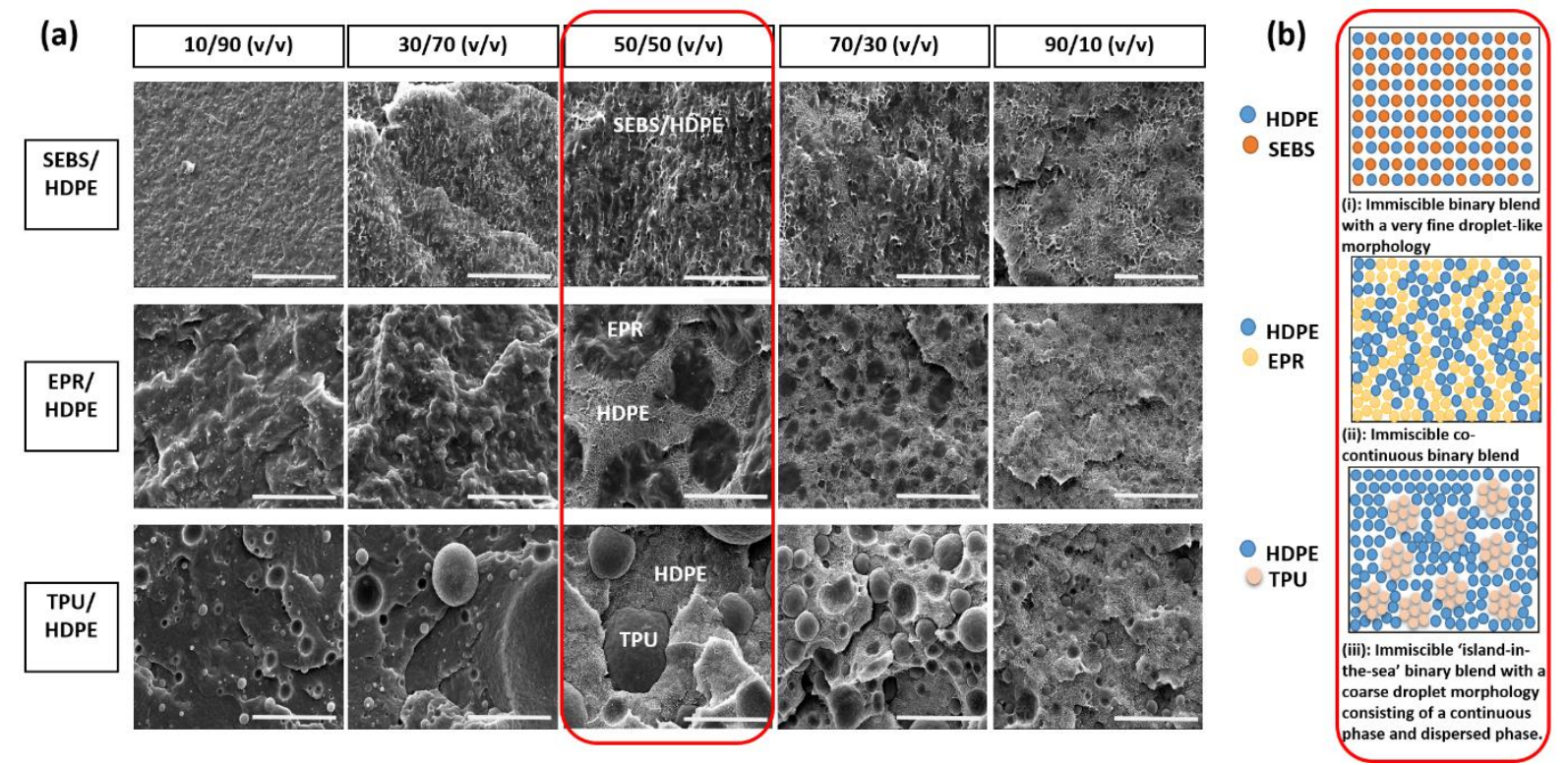

Fig. 1 (a) Morphologies of the three TPE/HDPE blends at different blend compositions (SEBS/HDPE, EPR/HDPE, and TPU/HDPE at 10/90, 30/70, 50/50, 70/30 and 90/10 v/v). Scale bar, $20 \mathrm{~mm}$; (b) schematic illustration of three distinct types of polymer blends at around $50 / 50 \mathrm{v} / \mathrm{v}$.

\section{Prediction of selective localization of GNPs in polymer blends}

In order to assess the influence of the different blend morphologies, as observed in the previous section on the selective localization behaviour of GNPs in these TPE/HDPE blends, a simple thermodynamic analytical model is used. The GNP location, either in one specific phase or at the interface of an immiscible polymer blend, is dictated by the minimisation of the interfacial surface energy. ${ }^{40-42}$ Based on Young's equation, the wetting coefficient $\left(\omega_{a}\right)$ can be theoretically deduced from eqn (1) as shown below: ${ }^{20}$

$\omega_{a}=\frac{\gamma_{\text {filler-polymer 1 }}-\gamma_{\text {filler-polymer } 2}}{\gamma_{\text {polymer } 1,2}}$

Where $\gamma_{\text {filler-polymer 1 }}, \gamma_{\text {filler-polymer } 2}$ and $\gamma_{\text {polymer 1,2 }}$ represent the interfacial energies between filler and polymer 1 , the filler and polymer 2 and between polymer 1 and polymer 2 , respectively. As described by Sumita et al. and afterwards by Fenouillot et al., values of $\omega_{a}>$ 
$1, \omega_{a}<-1$ or $-1<\omega_{a}<1$, mean that the filler will preferentially be localized in polymer 2 , polymer 1 or at the interface, respectively. ${ }^{27,} 29$ The interfacial energy can be estimated by a geometric mean equation (Eqn (2)):

$\gamma_{12}=\gamma_{1}+\gamma_{2}-2\left(\sqrt{\gamma_{1}^{\mathrm{d}} \gamma_{2}^{\mathrm{d}}}+\sqrt{\gamma_{1}^{\mathrm{p}} \gamma_{2}^{\mathrm{p}}}\right)$

Where $\gamma_{1}$ and $\gamma_{2}$ are the surface energies of components 1 and $2 ; \gamma_{1}^{\mathrm{d}}$ and $\gamma_{2}^{\mathrm{d}}$ are the dispersive parts of the surface energies of component 1 and $2 ; \gamma_{1}^{\mathrm{p}}$ and $\gamma_{2}^{\mathrm{p}}$ are the polar parts of the surface energies of components 1 and $2 .^{20}$

The surface energies of the pure polymers can be calculated by the model of Owens, Wendt, Rabel and Kaelble (OWRK model), which considers the geometric mean of the dispersive and polar parts of the liquid's surface energy and of the solid's surface energy, in combination with the Young's equation. ${ }^{43}$ It is worth noting that this prediction was based on the surface energy measured at room temperature rather than at the mixing temperature. Although absolute values of surface energies would be different at higher, relative values between different polymers would still be maintained.

Table 3 shows the tested contact angles of HDPE, SEBS, EPR and TPU films, and the corresponding calculated surface energies. Based on the surface energy data, the corresponding interfacial energy is calculated (see ESI, $\uparrow$ Table S1). According to the data of interfacial energy and eqn (1), $\omega_{a}$ are listed for the GNP filled polymer blends respectively. The properties of the GNP/HDPE masterbatch have also been taken into consideration for predicting the final location of the GNPs. Following this theoretical approach, the thermodynamic calculations indicated that the GNPs should remain in the HDPE phase in the case of EPR/HDPE blends, while in SEBS/HDPE as well as TPU/HDPE, the GNPs should be preferentially located at the interface between the two polymer phases (Table 4). 
Table 3 Surface energy data of the different components

\begin{tabular}{|c|c|c|c|c|c|}
\hline \multirow{2}{*}{ Material } & \multicolumn{2}{|c|}{$\theta$ [deg] } & \multicolumn{3}{|c|}{ Surface energy $\left[\mathrm{mJ} \mathrm{m}^{-2}\right]$} \\
\hline & Water & Ethylene Glycol & $\gamma$ & $\gamma^{\mathrm{d}}$ & $\gamma^{\mathrm{p}}$ \\
\hline HDPE & 95.4 & 71.7 & 30.0 & 29.4 & 0.6 \\
\hline SEBS & 94.2 & 83.1 & 15.9 & 4.0 & 11.9 \\
\hline EPR & 94.5 & 69.0 & 34.5 & 34.2 & 0.3 \\
\hline TPU & 99.9 & 82.0 & 18.4 & 16.5 & 1.8 \\
\hline GNP & - & - & 53.0 & 39.1 & $13.9^{44}$ \\
\hline
\end{tabular}

Table 4 Wetting coefficient and predicted location of GNPs

\begin{tabular}{lcc}
\hline Blends & $\begin{array}{c}\boldsymbol{\omega}_{\boldsymbol{a}} \\
\text { Geometric mean equation }\end{array}$ & Predicted location \\
\hline GNP/(SEBS/HDPE) & -0.4517 & SEBS/HDPE interface \\
GNP/(EPR/HDPE) & -4.2280 & HDPE phase \\
GNP/(TPU/HDPE) & -0.3742 & TPU/HDPE interface \\
\hline
\end{tabular}

\section{Morphology of GNP filled binary polymer blends}

In order to get an insight into the microstructure of the composites and the preferential localization of the GNPs in the blends, SEM micrographs are presented in Fig. 2. Morphologies of HDPE/GNP (12 wt\%) composites are shown in Fig. 2a as a reference for the GNP distribution in the three TPE/HDPE blends.

Fig. $2 b$ illustrates the morphology of the fine morphology blend SEBS/HDPE/GNP (50 wt\% SEBS), where the GNP dispersion condition is very similar to the case of the HDPE/GNP composite. In theory, the fillers should be located at the interface between the two polymers. However, due to the fine morphology of the SEBS/HDPE blend, the size of the SEBS phases is much smaller than that of the GNP. Hence, on the scale of the SEM micrographs, it is reasonable to assume that the GNPs are dispersed homogeneously in the SEBS/HDPE blend. 
In the co-continuous EPR/HDPE/GNP blend (50 wt\% EPR), the GNP particles are mostly present in the HDPE phase rather than in the EPR phase (Fig. 2c), which is in accordance with the prediction by the wetting coefficient. Moreover, the higher viscosity of EPR (see the ESI, $\uparrow$ Fig. S1) is an additional factor which explains why the fillers stay in HDPE, and is in agreement with Feng et al. and Zhou et al. ${ }^{45,46}$ Fig. $2 \mathrm{~d}$ shows the TPU/HDPE/GNP blends (50 wt\% TPU) whose morphology differs from the neat TPU/HDPE blends with the same composition. This indicates that the addition of fillers influences the blend morphology, which makes the composites forming a less clear droplet shaped morphology. ${ }^{25}$ It can be found that a large proportion of the GNP fillers are located at the interface between the HDPE and TPU phase, while still a smaller proportion of the GNPs in both the HDPE and TPU phase. Apparently due to the relatively large lateral dimensions of GNP fillers, some of the fillers at interfacial regions are bridging two phases, resulting in a less distinguished two phase morphology which is slightly different from previous observations in pure polymer binary systems (higher magnification SEM graphs of the three blends can be found in the ESI, $\uparrow$ Fig. S2). The location of GNPs and the morphologies of the polymer blends should have a significant influence on the electrical and pyroresistive properties of the composites as will be discussed below. 



Fig. 2 SEM images of (a) HDPE/GNP (12 wt $\%$ ) composites as a reference for GNP distribution;

(b) SEBS/HDPE/GNP (50 wt\% SEBS), with GNPs dispersed similar to the HDPE/GNP composite; (c) EPR/HDPE/GNP (50 wt\% EPR), with the GNP particles mostly present in the HDPE phase rather than in the EPR phase; (d) TPU/HDPE/GNP (50 wt\% TPU), with a large proportion of the GNP fillers located near the interface between HDPE and TPU phase.

\section{Electrical properties of GNP filled polymer blends}

The volume electrical conductivity of HDPE/GNP composites made by melt-compounding and compression moulding are displayed in Fig. 3a. Classical percolation theory is used to describe the conductivity change of the HDPE/GNP composite by a scaling law:

$\sigma=\sigma_{0}\left(\varphi-\varphi_{c}\right)^{t}$ 
Where $\sigma$ is the conductivity of the conductive polymer composite (CPC), $\sigma_{0}$ is a scaling factor, $\varphi$ is the filler content and $\varphi_{c}$ is the percolation threshold, and $\mathrm{t}$ is the critical exponent which is expected to depend on the conductive system dimensionality only. The percolation threshold can be determined by fitting the experimental data with eqn (3). In the case of HDPE/GNP composites, the percolation threshold is calculated to be $8.8 \mathrm{wt} \%$ (4.0 vol\%). (Fit shown in the inset.)

It is interesting to evaluate the dependence of the conductivity on the blend composition. By blending $10,20,35$, and $50 \mathrm{wt} \%$ of a secondary thermoplastic elastomeric phase into the HDPE/GNP masterbatch (24 wt $\%$ of GNP loading), the electrical conductivity evolves in quite a different way, depending on the TPE selected. SEBS/HDPE/GNP composites show the largest drop in the conductivity level with the addition of SEBS, as shown in Fig. 3b. The conductivity of $50 \mathrm{wt} \%$ SEBS is around 5 orders of magnitude lower than the HDPE/GNP masterbatch. On the other hand, the conductivity of $50 \mathrm{wt} \%$ EPR is only reduced by one order of magnitude, which means that here the conductive pathways are barely affected by the addition of the secondary polymer. The conductivity change of the TPU/HDPE/GNP composite sits in between that of the other two blends.

The above conductivity results confirmed the morphological microstructures of the three blends and the preferential localization of the GNP in each blend. Due to the good dispersion of GNPs in the highly compatible SEBS/HDPE blend, a typical dilution effect is observed. While in the EPR/HDPE binary blends, the fillers stay in the HDPE phase within the cocontinuous structure. Thus, the filler content in the HDPE phase stays at a relatively same level as the masterbatch loading. In the TPU/HDPE/GNP composite, most of the GNPs move to the interface between TPU and HDPE, while a smaller proportion of the filler migrates into the TPU phase, which results in a reduced conductivity, albeit to a lesser degree as for the 
SEBS/HDPE/GNP blends. The conductivity behaviour of the composites should strongly influence the pyroresistive behaviour of the blend systems, which will be discussed in the next section.
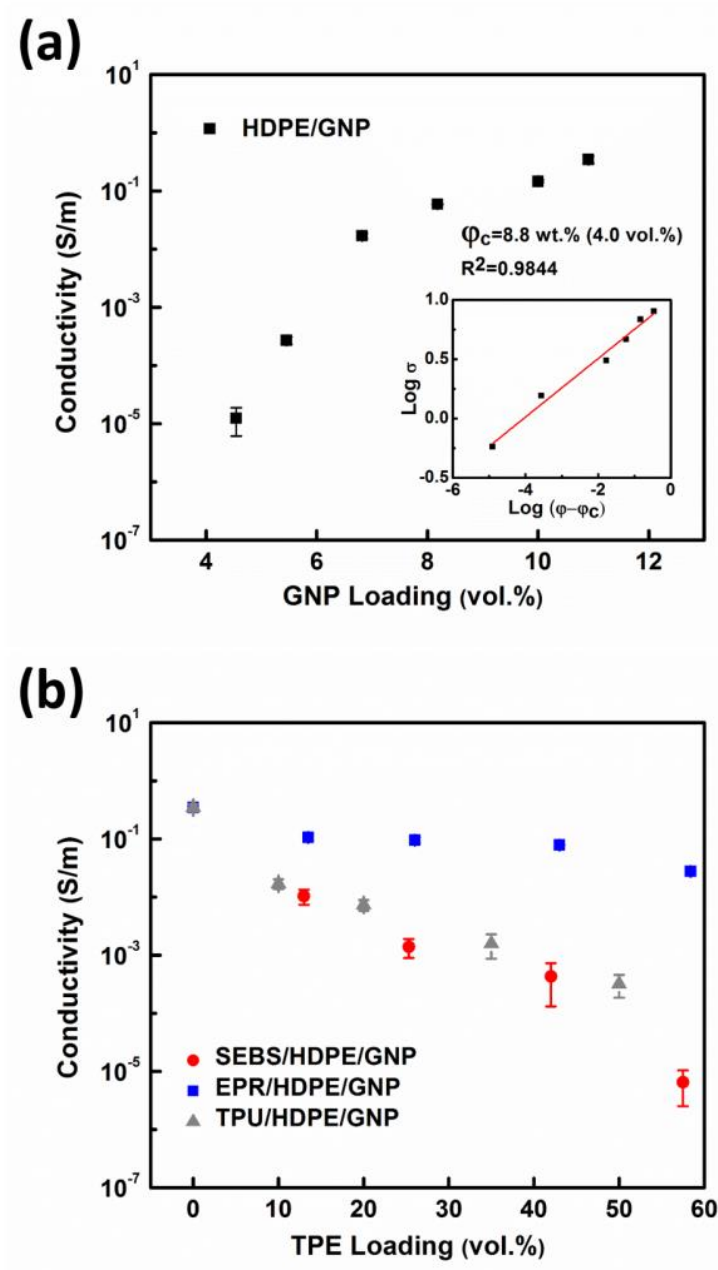

Fig. 3 (a) Percolation curves of HDPE/GNP composites and percolation threshold (jc) of 4.0 vol\%, calculated by fitting the experimental data with eqn (3) (see the inset), and (b) dependence of conductivity on the ratio of binary TPE polymer for SEBS/HDPE/GNP; EPR/HDPE/GNP, and TPU/HDPE/GNP composites.

\section{Pyroresistive behaviour of GNP filled polymer blends}

The effect of temperature on the electrical resistivity of the neat HDPE/GNP composites is shown in Fig. 4a. The resistivity barely changes with temperature till $130{ }^{\circ} \mathrm{C}$, after which a 
very sharp increase is experienced up to 140 to $150{ }^{\circ} \mathrm{C}$, demonstrating a large PTC effect. This critical temperature mainly depends on the melting temperature of HDPE as indicated by the DSC curve of pure HDPE (see ESI, $\uparrow$ Fig. S3). This can be explained as follows: at relatively low temperatures $\left(<120^{\circ} \mathrm{C}\right)$, thermal expansion of the polymer matrix reduces the contact between the GNP particles only slightly, leading to a gradual increase in resistivity of the nanocomposite. However, around the melting temperature of HDPE (120 to $\left.140{ }^{\circ} \mathrm{C}\right)$, a huge increase in thermal expansion due to melting of the crystalline phase disconnects the conducting paths, resulting in an abrupt jump in resistivity of more than 2 orders of magnitude. At temperature well above the melting point of HDPE, the resistivity of the composite begins to decrease, leading to a negative temperature coefficient (NTC) effect. This can be ascribed to 'dynamic percolation' or the formation of new conductive pathways resulting from the relaxation of the polymer structure and the agglomeration of GNPs when the melt viscosity of HDPE is sufficiently low. $5,47,48$ Moreover, the lower the GNP content, the higher the initial resistivity as well as the PTC intensity value. ${ }^{49}$

The addition of a secondary thermoplastic elastomeric phase significantly modified the PTC behaviour of the HDPE/ GNP composites. The PTC effect of SEBS/HDPE/GNP blends shows a similar trend as of HDPE/GNP composites at each blend composition, although the room temperature resistivity is significantly affected by the addition of SEBS. However, the PTC intensity is almost invariant with the SEBS loading (Fig. 4b). On the other hand, both the room temperature resistivity and the PTC intensity of the EPR/HDPE/GNP blends are independent of EPR loading as shown in Fig. 4c. This result is consistent with the morphological SEM observations, which showed a continuous HDPE phase where GNPs are preferentially located. This dominates the electrical and pyroresistive behaviour of the blends, even at higher EPR loadings. In the case of the TPU/HDPE/GNP blends (Fig. 4d), the migration of GNP towards the HDPE/TPU interface and into the TPU phase resulted in an increase in room temperature 
resistivity with TPU content while the PTC intensity decreased. It can therefore be concluded that tuneable self-regulating heating functions can be achieved by controlling (i) the blend morphology, (ii) the conductive filler network and (iii) the localization of the filler within the blend.

(a)

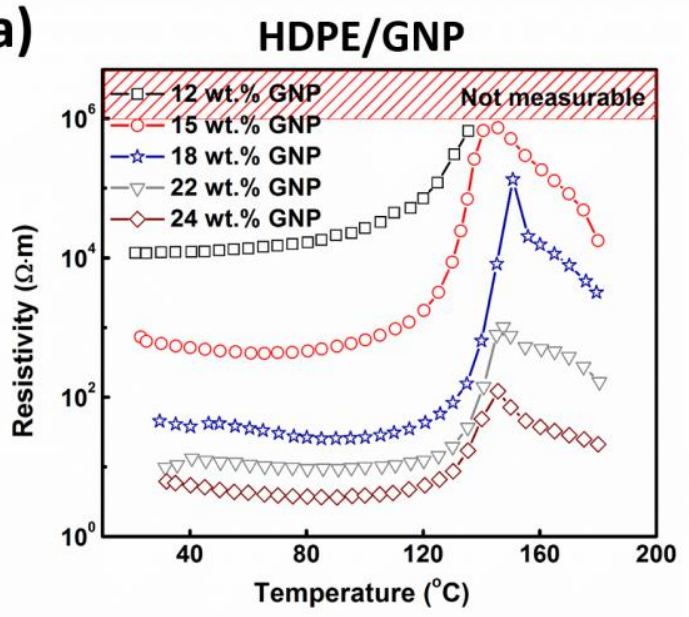

(c)

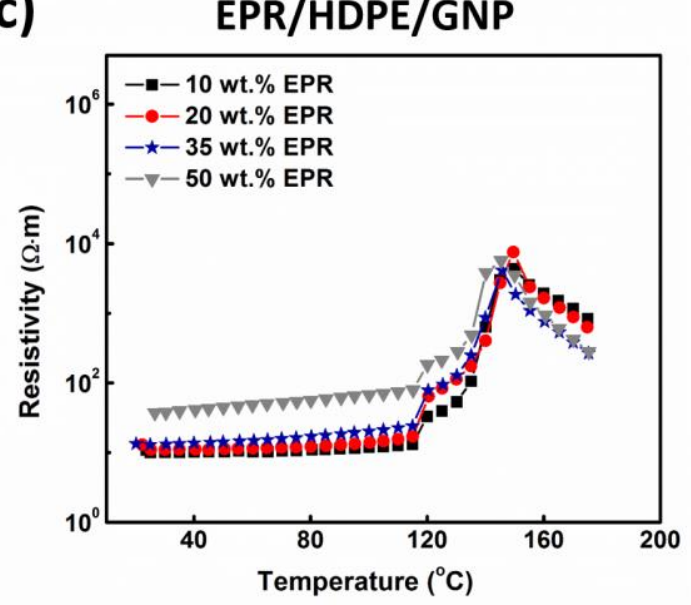

(b)

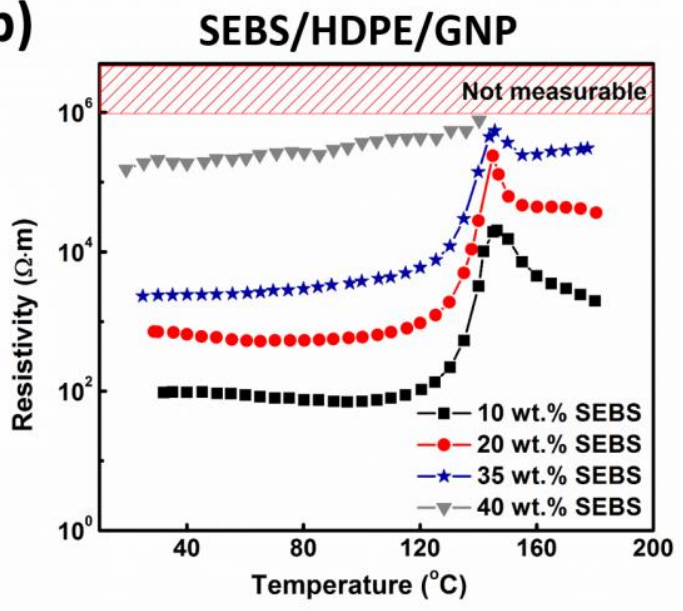

(d)

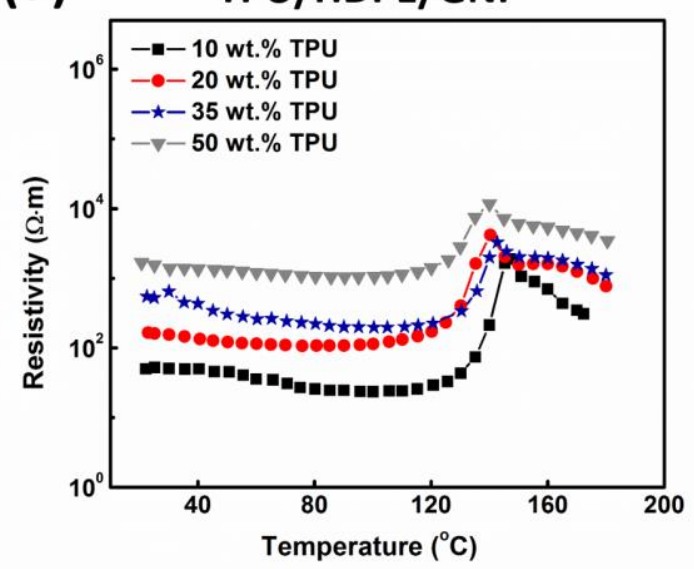

Fig. 4 The effect of temperature on the electrical resistivity of: (a) HDPE/GNP composites with different filler content; (b) SEBS/HDPE/GNP composites with different SEBS loadings, where room temperature resistivity is significantly affected by the addition of SEBS; (c) EPR/HDPE/GNP composites with different EPR loadings, where both room temperature resistivity and PTC intensity were independent of EPR content; and (d) TPU/HDPE/GNP 
composites with different TPU contents, where an increase in room temperature resistivity is observed with TPU content while the PTC intensity decreased.

\section{Joule heating properties of GNP filled blends}

Efficient Joule heating is an essential requirement in a final self-regulating heating device. The electrical heating properties of HDPE/GNP MB and TPE/HDPE/GNP composites (with a GNP content of about $19.2 \mathrm{wt} \%$ and a TPE content of $20 \mathrm{wt} \%$ respectively) are evaluated by measuring the changes in temperature and resistance under an applied AC voltage and are shown in Fig. 5a. For all four composites; HDPE/GNP, SEBS/HDPE/GNP, EPR/HDPE/GNP and TPU/HDPE/GNP, a stable self-regulating temperature between 100 and $120^{\circ} \mathrm{C}$ is achieved within a short period of time. Fig. $5 \mathrm{~b}$ shows the change in heating power, which is maximum at room temperature and autonomously decreases until the same temperature range is reached. Thermal images show the uniformly heated samples for each blend in Fig. 5c. 
(a)

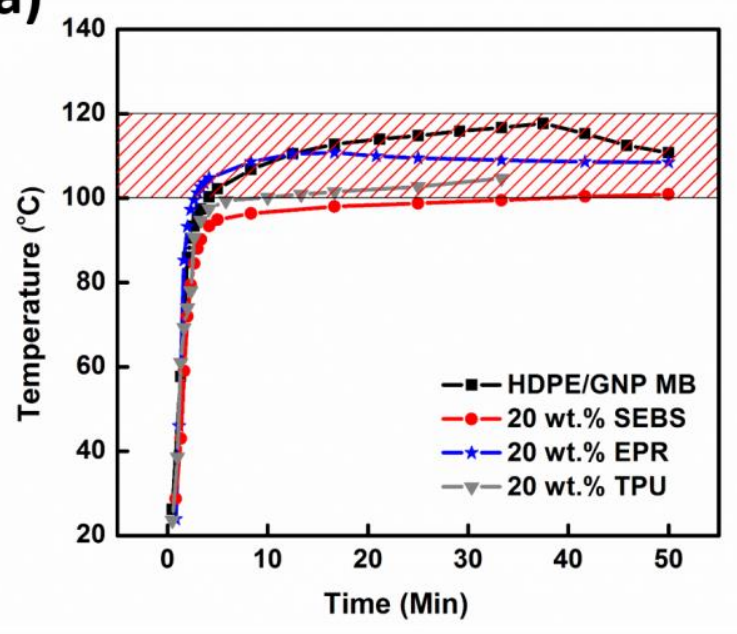

(b)

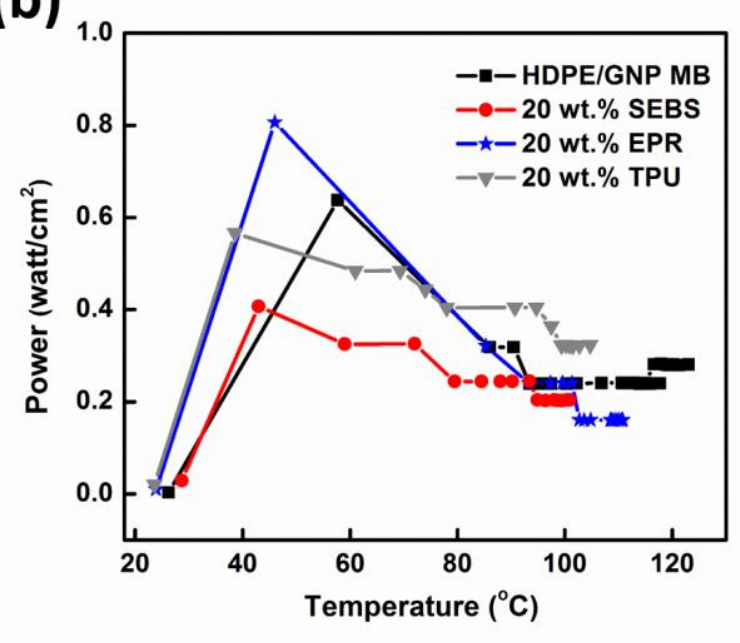

(c)
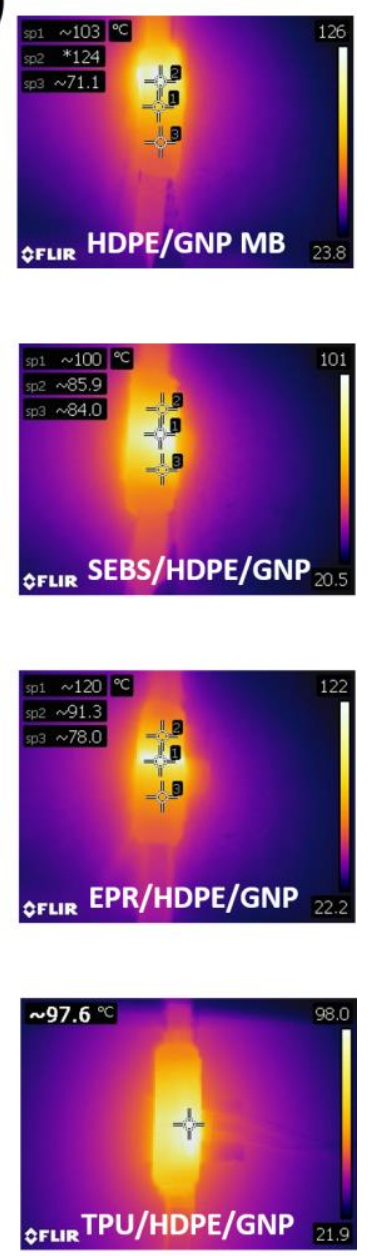

Fig. 5 (a) Stabilised self-regulating temperatures between 100 and $120^{\circ} \mathrm{C}$ were achieved by a Joule heating approach for each of the four composites (HDPE/GNP MB, SEBS/HDPE/GNP, EPR/HDPE/GNP and TPU/HDPE/GNP with a GNP loading of about $19.2 \mathrm{wt} \%$ and a TPE content of $20 \mathrm{wt} \%$ ); (b) electrical power changes with increasing temperature, autonomously decreases and maintains at a similar range when PTC temperature is reached; (c) thermal images show uniformly heated samples for each type of composite.

\section{Mechanical behaviour of GNP filled polymer blends}

In order to develop high performance GNP based composites, the effect of TPE addition on the mechanical tensile properties was examined on the HDPE/GNP masterbatch as well as for the 
TPE/HDPE/GNP composites. Typical stress-strain curves as a function of secondary TPE phase are shown in Fig. 6 and Young's modulus, tensile strength, and strain-at-break are reported (see ESI, $\uparrow$ Table S2). For SEBS/HDPE/GNP blends, the strain-at-break increases dramatically with increasing concentration of the thermoplastic elastomer, while the elastic modulus is inversely correlated as shown in the ESI, $\uparrow$ Table S2. Dynamic mechanical analysis (DMA) of a neat polymer has been demonstrated in the ESI, $\uparrow$ Fig. S4, showing the storage modulus as a function of temperature as a guidance of elastic performance of TPE. Blending with SEBS clearly reduces the brittleness of the highly loaded masterbatch. However, for the EPR/HDPE/GNP blends there is no clear trend of an increase in strain-at-break until $50 \mathrm{wt} \%$ EPR was incorporated into the masterbatch. Only when a co-continuous TPE phase is formed in the blend, a significant improvement in ductility is obtained for this system. In TPU/HDPE/GNP blends we even observed an adverse effect of the increasing TPE content on ductility. Here the strain-at-break decreases slightly at a high elastomer content. This is possibly due to partial phase segregation as a result of the poor interfacial bonding between the HDPE and TPU phases. 
(a)

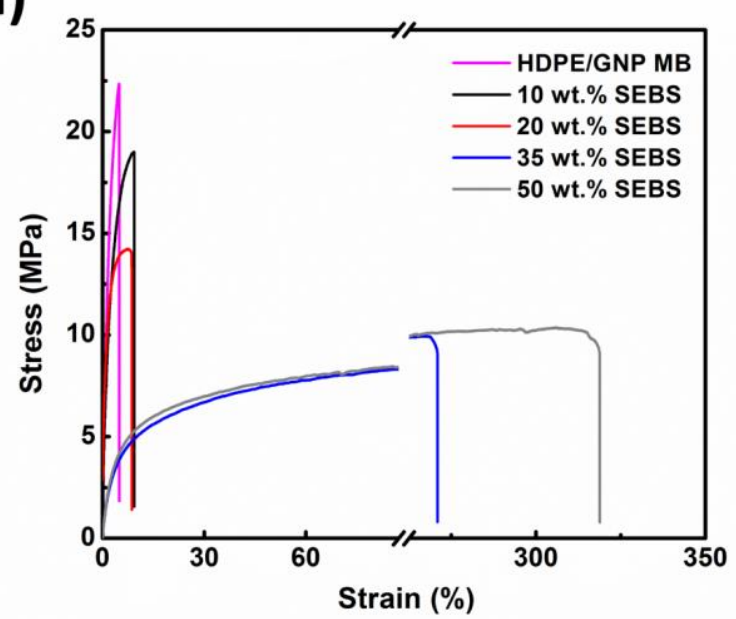

(b)

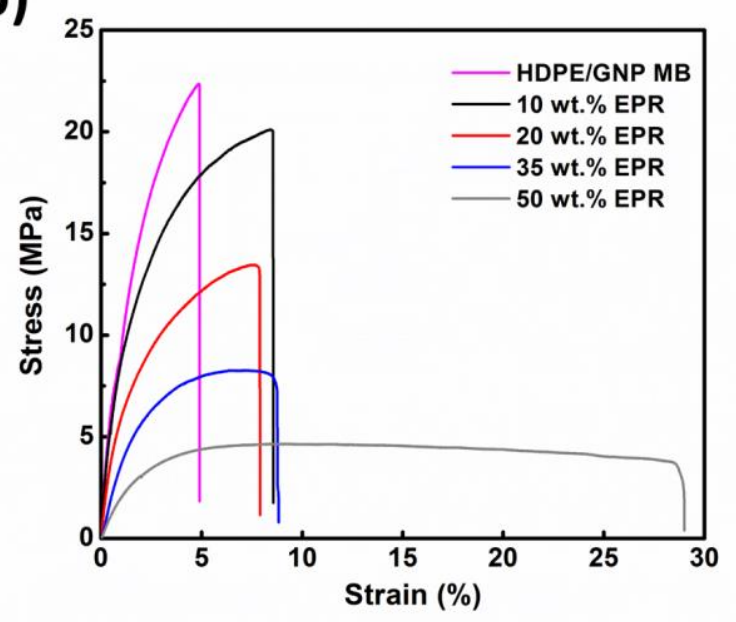

(c)

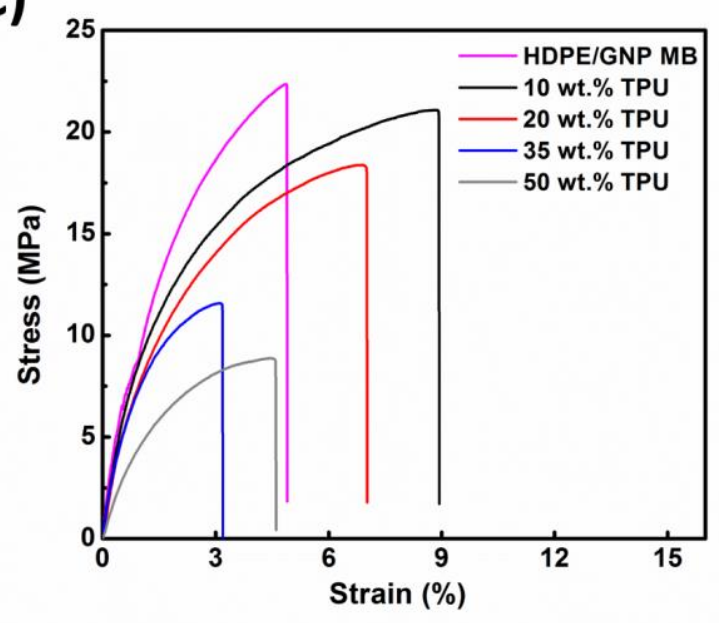

Fig. 6 Stress-strain responses of the HDPE/GNP and TPE/HDPE/GNP composites as a function of secondary TPE phase loading. 
For many heating devices such as wearable smart heaters and heating mats or jackets, high mechanical flexibility is another important requirement as these devices need to be wrapped around complex structures. For example, in order to wrap around canned food and heat up, the device requires a bending radius of at least $5 \mathrm{~cm}$. Unfortunately, the highly filled HDPE/GNP masterbatch is a rather brittle material (Fig. 7a), failing at a deflection of $1 \mathrm{~mm}$ in a three-point bending experiment, equivalent to a radius of curvature of $5 \mathrm{~cm}$. Fig. $7 \mathrm{~b}$ presents the actual photos of bending tests of composite samples, and demonstrates the flexibility and robustness of the GNP filled binary blends. The bending radius increased dramatically as shown in Fig. 7a, with the electrical resistance measured upon bending. The failure radius is reduced to $3 \mathrm{~cm}$ with $50 \mathrm{wt} \%$ TPU added, while EPR/HDPE/GNP blends decrease further the failure radius to $1 \mathrm{~cm}$. SEBS/HDPE/GNP blends show the greatest improvement in flexibility with a failure radius as low as $0.3 \mathrm{~cm}$, which is sufficient for most of the applications requiring high flexibility. Considering the morphological differences between the three TPE/HDPE blends, the EPR/HDPE/GNP blend shows the least variation in electrical resistance during bending, which can be largely attributed to the co-continuous blend morphology of this system and the preferential GNP localization in the continuous HDPE phase. SEBS/HDPE/GNP blends showed the greatest improved mechanical bending flexibility compared with other blends due to the fine blend morphologies. 


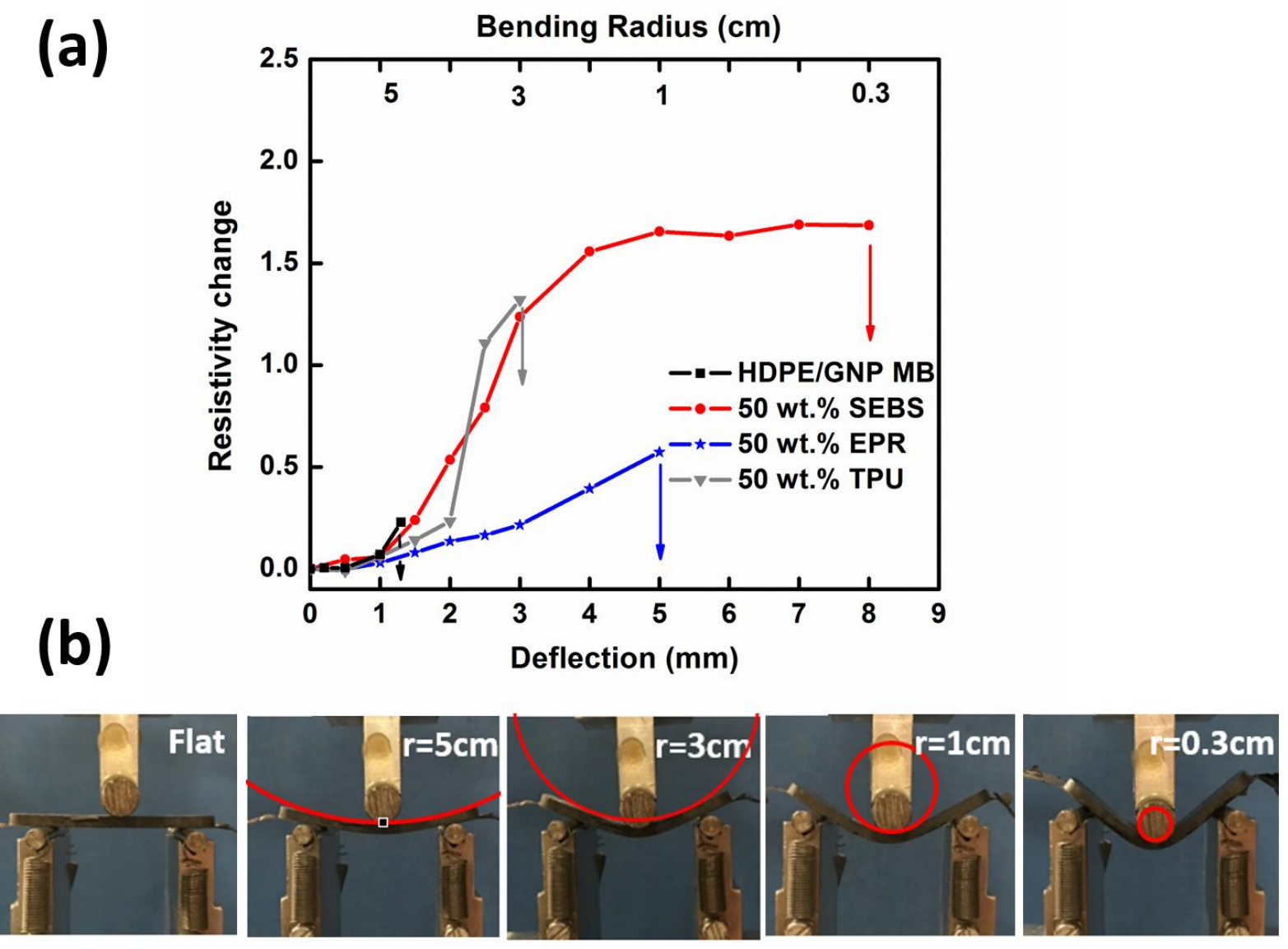

Fig. 7 Bending characterization of the HDPE/GNP MB and TPE/HDPE/GNP composites. (a) Electrical resistance change of samples as a function of deflection and bending radii, the addition of $50 \mathrm{wt} \%$ TPU, EPR, SEBS reduced the failure bending radius to $3 \mathrm{~cm}, 1 \mathrm{~cm}$ and 0.3 $\mathrm{cm}$, respectively; (b) photographs of SBES/HDPE/GNP (50 wt\% SEBS) composites at different bending radii, demonstrating the flexibility and robustness of the GNP filled binary blends.

\section{Conclusions}

The selection of a secondary thermoplastic elastomeric (TPE) phase to be added to a HDPE/GNP composite was demonstrated to greatly affect the morphology of the resulting blends, leading to an immiscible binary blend with either a fine or course droplet morphology, or a co-continuous morphology. The localization of the conductive nanofiller can be controlled 
to be either in one phase or at the interface between two phases, as predicted by a simple analytical model together with the Young's equation, depending on polymer viscosity and surface energies. The control over the microstructure enabled tunable electrical conductivity and PTC behaviour, with different initial room temperature resistivity and PTC intensity for each blend. Moreover, developed materials' flexibility was greatly enhanced for the TPE modified composites, as demonstrated by a 7 times increase in the failure bending deflection. Compared to the neat HDPE/GNP composites the strain-at-break values increased for the SEBS/HDPE/GNP blend from 5\% to more than 300\%. The EPR/HDPE/GNP blend, with a cocontinuous morphology, appears particularly interesting for future applications in selfregulating heating devices as the electrical resistivity is independent of the bending radius to a large extent.

\section{Conflicts of interest}

There are no conflicts to declare.

\section{Acknowledgements}

The authors gratefully acknowledge the financial support from LMK Thermosafe Ltd, and Innovate UK (KTP number: KTP009619).

\section{References}

1 H. Deng, L. Lin, M. Ji, S. Zhang, M. Yang and Q. Fu, Prog. Polym. Sci., 2014, 39, 627-655.

2 J. N. Coleman, U. Khan, W. J. Blau and Y. K. Gun'ko, Carbon, 2006, 44, 1624-1652.

3 Y. Liu, H. Zhang, H. Porwal, W. Tu, J. Evans, M. Newton, J. J. Busfield, T. Peijs and E. Bilotti, Adv. Funct. Mater., 2017, 27, 1702253.

4 E. T. Thostenson and T. W. Chou, Adv. Mater., 2006, 18, 2837-2841.

5 E. Bilotti, R. Zhang, H. Deng, M. Baxendale and T. Peijs, J. Mater. Chem., 2010, 20, 94499455.

6 T. Villmow, S. Pegel, A. John, R. Rentenberger and P. Potschke, Mater. Today, 2011, 14, $340-345$. 
7 H. Zhang, E. Bilotti and T. Peijs, Nanocomposites, 2015, 1, 167-184.

8 H. Zhang, Y. Liu, E. Bilotti and T. Peijs, Adv. Compos. Lett., 2015, 24, 92-97.

9 J. Jeon, H. B. R. Lee and Z. Bao, Adv. Mater., 2013, 25, 850-855.

10 K. Ohe and Y. Naito, Jpn. J. Appl. Phys., 1971, 10, 99.

11 H. Xu, Y. Wu, D. Yang, J. Wang and H. Xie, Rev. Adv. Mater. Sci., 2011, 27, 173-183.

12 J.-W. Zha, D.-H. Wu, Y. Yang, Y.-H. Wu, R. K. Li and Z.-M. Dang, RSC Adv., 2017, 7, $11338-11344$.

13 J. Feng and C.-M. Chan, Polymer, 2000, 41, 4559-4565.

14 H. Xu, Z. Dang, D. Shi and J. Bai, J. Mater. Chem., 2008, 18, 2685-2690.

15 L. Shen, F. Q. Wang, H. Yang and Q. R. Meng, Polym. Test., 2011, 30, 442-448.

16 G. Yu, Q. Zhang, H. M. Zeng, Y. H. Hou and H. B. Zhang, Polym. Eng. Sci., 1999, 39, $1678-1688$.

17 A. Rybak, G. Boiteux, F. Melis and G. Seytre, Compos. Sci. Technol., 2010, 70, 410-416.

18 L. Lin, S. Liu, Q. Zhang, X. Li, M. Ji, H. Deng and Q. Fu, ACS Appl. Mater. Interfaces, $2013,5,5815-5824$.

19 B. Li, Y. Zhang, Z. Li, S. Li and X. Zhang, J. Phys. Chem. B, 2010, 114, 689-696.

20 J. W. Zha, W. K. Li, R. J. Liao, J. B. Bai and Z. M. Dang, J. Mater. Chem. A, 2013, 1, 843851.

21 E. Asare, A. Basir, W. Tu, H. Porwal, H. Zhang, Y. Liu, J. Evans, M. Newton, T. Peijs and E. Bilotti, Nanocomposites, 2016, 2, 58-64.

22 E. Bilotti, H. Zhang, H. Deng, R. Zhang, Q. Fu and T. Peijs, Compos. Sci. Technol., 2013, $74,85-90$.

23 Z. Chen, P. C. Hsu, J. Lopez, Y. Li, J. W. F. To, N. Liu, C. Wang, S. C. Andrews, J. Liu, Y. Cui and Z. Bao, Nat. Energy, 2016, 1, 15009.

24 P. Potschke and D. Paul, J. Macromol. Sci., Part C: Polym. Rev., 2003, 43, 87-141.

25 A. Nuzzo, E. Bilotti, T. Peijs, D. Acierno and G. Filippone, Polymer, 2014, 55, 4908-4919.

26 N. Meng, X. Zhu, R. Mao, M. J. Reece and E. Bilotti, J. Mater. Chem. C, 2017, 5, 32963305 .

27 F. Fenouillot, P. Cassagnau and J.-C. Majeste, Polymer, 2009, 50, 1333-1350.

28 C. Zhang, X. Yi, H. Yui, S. Asai and M. Sumita, Mater. Lett., 1998, 36, 186-190.

29 M. Sumita, K. Sakata, S. Asai, K. Miyasaka and H. Nakagawa, Polym. Bull., 1991, 25, 265271.

30 S. Asai, K. Sakata, M. Sumita and K. Miyasaka, Polym. J., 1992, 24, 415-420.

31 B. Chen, X. Li, S. Xu, T. Tang, B. Zhou and B. Huang, Polymer, 2002, 43, 953-961.

32 S. Tjong and S. Xu, J. Appl. Polym. Sci., 1998, 68, 1099-1108. 
33 Z. S. Petrovic, J. Budinski-Simendic, V. Divjakovic and Z. Skrbic, J. Appl. Polym. Sci., 1996, 59, 301-310.

34 P. Potschke, K. Wallheinke and H. Stutz, Polym. Eng. Sci., 1999, 39, 1035-1048.

35 P. Potschke, K. Wallheinke, H. Fritsche and H. Stutz, J. Appl. Polym. Sci., 1997, 64, 749762.

36 E. Bilotti, H. Porwal, Y. Liu, M. Newton and J. Evans, International Patent Application No. PCT/GB2017/051909. Filed on 30 June 2016. (Claiming priority from GB Patent Application No. 1611397.9), 2016, Applicant: LMK Thermosafe Ltd.

37 M. Fang, K. Wang, H. Lu, Y. Yang and S. Nutt, J. Mater. Chem., 2009, 19, 7098-7105.

38 J. Li, P. L. Ma and B. D. Favis, Macromolecules, 2002, 35, 2005-2016.

39 A. Chirawithayaboon and S. Kiatkamjornwong, J. Appl. Polym. Sci., 2004, 91, 742-755.

40 A. Goldel, G. Kasaliwal and P. Potschke, Macromol. Rapid Commun., 2009, 30, 423-429.

41 A. Goldel, A. Marmur, G. R. Kasaliwal, P. Potschke and G. Heinrich, Macromolecules, 2011, 44, 6094-6102.

42 Y. Lan, H. Liu, X. Cao, S. Zhao, K. Dai, X. Yan, G. Zheng, C. Liu, C. Shen and Z. Guo, Polymer, 2016, 97, 11-19.

43 D. K. Owens and R. Wendt, J. Appl. Polym. Sci., 1969, 13, 1741-1747.

44 A. Kozbial, Z. Li, C. Conaway, R. McGinley, S. Dhingra, V. Vahdat, F. Zhou, B. D’Urso, H. Liu and L. Li, Langmuir, 2014, 30, 8598-8606.

45 P. Zhou, W. Yu, C. X. Zhou, F. Liu, L. M. Hou and J. Wang, J. Appl. Polym. Sci., 2007, $103,487-492$.

46 J. Feng and C.-M. Chan, Polymer, 2000, 41, 7279-7282.

47 H. Deng, T. Skipa, R. Zhang, D. Lellinger, E. Bilotti, I. Alig and T. Peijs, Polymer, 2009, 50, 3747-3754.

48 H. Deng, T. Skipa, E. Bilotti, R. Zhang, D. Lellinger, L. Mezzo, Q. Fu, I. Alig and T. Peijs, Adv. Funct. Mater., 2010, 20, 1424-1432.

49 E. Asare, J. Evans, M. Newton, T. Peijs and E. Bilotti, Mater. Des., 2016, 97, 459-463. 